\title{
Psychogenic nonepileptic seizures: a treatment review. What have we learned since the beginning of the millennium?
}

This article was published in the following Dove Press journal:

Neuropsychiatric Disease and Treatment

6 December 2012

Number of times this article has been viewed

\section{Gaston Baslet}

Department of Psychiatry, Brigham and Women's Hospital, Harvard Medical School, Boston, MA, USA
Correspondence: Gaston Baslet Department of Psychiatry, Brigham and Women's Hospital, 22I Longwood Avenue, Boston, MA 02115, USA

Tel +l 6177324818

Fax +I 6177388703

Email gbaslet@partners.org
Abstract: Psychogenic nonepileptic seizures (PNES) can significantly affect an individual's quality of life, the health care system, and even society. The first decade of the new millennium has seen renewed interest in this condition, but etiological understanding and evidence-based treatment availability remain limited. After the diagnosis of PNES is established, the first therapeutic step includes a presentation of the diagnosis that facilitates engagement in treatment. The purpose of this review is to present the current evidence of treatments for PNES published since the year 2000 and to discuss further needs for clinical treatment implementation and research. This article reviews clinical trials that have evaluated the efficacy of structured, standardized psychotherapeutic and psychopharmacological interventions. The primary outcome measure in clinical trials for PNES is event frequency, although it is questionable whether this is the most accurate indicator of functional recovery. Cognitive behavioral therapy has evidence of efficacy, including one pilot randomized, controlled trial where cognitive behavioral therapy was compared with standard medical care. The antidepressant sertraline did not show a significant difference in event frequency change when compared to placebo in a pilot randomized, double-blind, controlled trial, but it did show a significant pre- versus posttreatment decrease in the active arm. Other interventions that have shown efficacy in uncontrolled trials include augmented psychodynamic interpersonal psychotherapy, group psychodynamic psychotherapy, group psychoeducation, and the antidepressant venlafaxine. Larger clinical trials of these promising treatments are necessary, while other psychotherapeutic interventions such as hypnotherapy, mindfulness-based therapies, and eye movement desensitization and reprocessing may deserve exploration. Flexible delivery of treatment that considers the heterogeneous backgrounds of patients is emphasized as necessary for successful outcomes in clinical practice.

Keywords: conversion disorder, therapeutics, clinical trials, psychotherapeutic interventions, psychopharmacological interventions

\section{Introduction}

Psychogenic nonepileptic seizures (PNES) are sudden, involuntary seizure-like attacks that, unlike epileptic seizures, are not related to electrographic ictal discharges. PNES presenting symptoms involve a wide array of nervous system functions, including changes in behavior, motor activity, sensation, cognitive, and autonomic functions.

PNES can be initially mistaken as epileptic seizures, and an accurate diagnosis is usually delayed by an average of 7 years. ${ }^{1}$ Diagnosis is usually confirmed via videoelectroencephalography ( $\mathrm{v}-\mathrm{EEG}$ ) monitoring in about a quarter of patients referred to epilepsy referral centers. ${ }^{2}$ There are no population-based studies to determine the incidence of this disorder, but it has been estimated that 300,000-400,000 people may suffer from PNES in the United States alone. ${ }^{3}$ PNES can greatly affect a patient's 
quality of life ${ }^{4,5}$ and are associated with high medical utilization rates and hence also high personal and societal costs. ${ }^{3}$ New evidence seems to indicate a higher premature mortality rate in PNES subjects than that found in a comparison with the Scottish general population, although deaths were not seizure related. ${ }^{6}$

Despite the recognition of this condition and its effect on individuals, society, and the health care system, currently there are significant limitations in the etiological understanding of PNES. Traditionally, PNES have been linked to a dysfunction in the processing of psychosocial stress. ${ }^{7}$ A conceptual framework has been proposed that explains PNES as involuntary, stimulus-driven behavioral responses facilitated by an orienting tendency where cognitive, emotional, and sensorimotor systems are not seemingly integrated. ${ }^{8}$ This response tendency may take place because of a number of vulnerability traits that increase a predisposition toward PNES, including dissociative tendencies, alexithymia, cognitive inflexibility, and hypervigilance, to name a few. ${ }^{8}$ This conceptual framework remains hypothetical and highlights the lack of a single etiological model that explains this phenomenon. ${ }^{9}$

Once PNES are properly diagnosed, treatment disposition usually includes a referral to mental health specialists. ${ }^{10}$ Traditionally, mental health providers willing to work with this population have utilized a variety of personalized psychotherapeutic approaches that address specific psychiatric comorbidities and hypothesized mechanisms. ${ }^{11}$ This may be necessary in part because of the heterogeneous psychiatric and medical backgrounds usually seen in the PNES

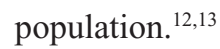

Guidelines on how to treat this complex population do not exist, owing to a lack of large, controlled trials evaluating the efficacy of different treatment modalities. ${ }^{14}$ In 2007 a Cochrane review looking at existing evidencebased treatments for PNES showed an alarming lack of randomized, controlled clinical trials with solid evidence of efficacy. ${ }^{15}$ According to a search of the PubMed database conducted for the present article, there were no clinical trials of specific interventions designed for PNES prior to 2000. Some follow-up studies undertaken prior to 2000 indicate that appropriate referral to mental health treatment had positive therapeutic effects. For instance, a follow-up study of 28 patients was published in 1999 and showed higher rates of event freedom and improvement in patients who received psychotherapy; however, the treatment delivered was not standardized among participants. ${ }^{16} \mathrm{~A}$ retrospective analysis of 61 PNES patients demonstrated higher rates of event freedom or reduction in those patients who received either psychotherapy or feedback and routine neurological care than in those who did not receive either. The psychotherapy treatment was provided either by a psychotherapist from a comprehensive epilepsy center or by community psychotherapists; no standardized treatment protocol is described to be followed beyond the diagnosis presentation. ${ }^{17}$ Remaining results consisted of isolated case reports for the adult population. ${ }^{18,19}$

The purpose of this review is to present the current evidence of treatments for PNES published since the year 2000 and to discuss further needs for clinical treatment implementation and research.

\section{What do we know about other PNES-related disorders?}

PNES subjects share many underlying psychopathological characteristics with other conversion and somatoform disorder patients. It remains uncertain what determines the final symptomatic expression of PNES. One study comparing motor conversion and PNES subjects found a higher incidence of adverse childhood experiences and life events in the PNES group, ${ }^{20}$ but it is far from clear if this constitutes a causative difference between patients who may have loss of consciousness as one of their manifestations, such as in PNES, and those with pure motor manifestations. Post-traumatic stress disorder (PTSD), other anxiety disorders, dissociative disorders, depression, and borderline personality disorder are comorbidities frequently encountered in PNES patients. ${ }^{21}$ Given the overlap in psychopathological structure with many of the disorders mentioned here, it is relevant to briefly review the current evidence of effective treatments for these disorders.

The strongest evidence for other conversion disorders comes from two randomized, controlled studies. In the first study, 20 patients with motor conversion symptoms received weekly sessions of hypnotherapy and the patients' change in conversion symptoms and level of disability were compared with a group of 24 subjects in a waiting list. Because the semiology of the motor conversion symptoms was mixed, two participants had PNES. Tremors, paresis, and gait complaints were common. Motor conversion symptoms, rated by the Video Rating Scale for Motor Conversion Symptoms, and level of disability both showed larger post- versus pretreatment improvement in the active group than in the control group. Subjects did not receive other psychotherapy interventions and there were no medication changes during the study, although nearly half of the subjects were on psychotropic medications at the onset of the trial..$^{22}$ 
In the second randomized, controlled trial, conducted by the same research group, 24 inpatients with motor conversion symptoms received eight weekly hypnotherapy sessions and were compared with 21 inpatients also with motor conversion symptoms but who received a supportive individual intervention instead during the admission. All subjects received multidisciplinary group-centered psychotherapy interventions and physical therapy. Eight subjects had PNES. Both groups showed post- versus pretreatment reductions in motor conversion symptoms and level of disability, with hypnotherapy providing no additional effect. ${ }^{23}$

Evidence-based treatment for other conversion disorders has also been demonstrated by uncontrolled trials; particularly, physical exercise ${ }^{24}$ and psychodynamic psychotherapy ${ }^{25}$ improve symptom severity in psychogenic movement disorders. Antidepressant medication showed reduction and even remission of psychogenic movements if the movements were not accompanied by other somatoform disorders such as hypochondriasis or somatization disorder. ${ }^{26}$ Repetitive transcranial magnetic stimulation over the motor cortex showed improvement in 62 of 70 patients (89\%) with psychogenic paralysis, with total recovery observed in $53(76 \%)$ of those subjects, although this was a retrospective review instead of a prospective trial. ${ }^{27}$

Two of the studies mentioned earlier for conversion disorder $^{22,23}$ included PNES subjects, but as these patients were mixed with other conversion patients and represented a minority of subjects within the studies, they were included in this section instead of in the PNES-specific review section.

Randomized, controlled trials of cognitive behavioral therapy (CBT) in somatization and specific-symptom syndromes (such as chronic fatigue syndrome, irritable bowel syndrome, chronic pain) have supported the effectiveness of this intervention. ${ }^{28}$ Psychodynamic interpersonal psychotherapy has also shown efficacy in irritable bowel syndrome. ${ }^{29,30}$ Antidepressants of various classes have also demonstrated reduction in medically unexplained symptoms including headache, fibromyalgia, functional gastrointestinal syndromes such as irritable bowel syndrome, idiopathic pain, tinnitus, and chronic fatigue. ${ }^{30,31}$

A review of the literature examining treatments for dissociative disorders such as dissociative identity disorder, depersonalization disorder, and dissociative disorder not otherwise specified shows a lack of standardized and welldesigned studies. ${ }^{32}$ One controlled single case study did show improvement in dissociative pathology in a dissociative identity disorder patient with cognitive analytic therapy. ${ }^{33}$
No other controlled studies have been published for dissociative disorders. It is possible that the need for long-term outcome studies may limit the utility of short-term interventions that can be adapted into a randomized, controlled trial in this population. ${ }^{32}$

A review of evidence-based treatments for other PNESrelated primary psychiatric disorders such as PTSD or borderline personality disorder is beyond the scope of this review. Nonetheless, it is noteworthy that there is mounting evidence of effective structured psychotherapies, with the most extensively studied psychotherapies being CBT for PTSD and other anxiety disorders ${ }^{34}$ and dialectical behavioral therapy for borderline personality disorder, ${ }^{35}$ although other forms of psychotherapy (such as eye movement desensitization and reprocessing [EMDR] in PTSD, or mentalization-based therapy in borderline personality disorder) and psychopharmacological interventions have also been investigated. ${ }^{35-39}$

Awareness of effective therapies in these conditions may help customize treatment in PNES subjects with these frequent comorbidities. From a research perspective, therapies proven effective in these related pathologies may become candidate interventions worth exploring in PNES.

\section{Limitations in PNES clinical trials}

A number of obstacles have been identified as contributing to the difficulty in conducting large clinical trials in PNES. Some of these obstacles are intrinsic to PNES psychopathology, such as the tendency to present in crises but reject support when offered, emotional lability, and approachavoidance behavioral patterns. Other impairments are logistical in nature and include driving restrictions, other cognitive and physical limitations related to medical and neurological illnesses, and a wide range of comorbid neurologic and psychiatric comorbidities, including epilepsy, which may preclude enrollment based on the study. ${ }^{40}$

The fields of neurology and psychiatry have played a role in the lack of priority given to PNES treatment development. Neurologists and emergency physicians tend to dismiss PNES patients and may interpret the patients' events as voluntary fabrications. Psychiatrists and psychologists may not treat PNES patients for fear of missing epilepsy when patients have ongoing events. ${ }^{41}$ As a result, PNES may be considered an "orphan" disorder that neither field wants to own as a priority to develop therapeutic approaches for.

The lack of a single etiological model for PNES ${ }^{9,14}$ limits the selection of mechanism-specific interventions and hence also limits outcome measures. However, a variety of proposed etiological mechanisms (including avoidance, dissociation, 
personality structure, interpersonal factors, illness representation, biological vulnerabilities) may inform treatment choice and selection of outcome measures. ${ }^{9,42}$

Regardless of the etiological model that dictates the intervention, useful outcome measures in PNES clinical trials include event frequency or percentage of subjects who achieved event freedom, psychopathological measures, social and interpersonal functioning measures, medical utilization rates, and quality of life. ${ }^{9}$ Although event freedom is usually the primary outcome in PNES clinical trials, it has been established that the percentage of functional recovery did not differ based on event remission 4.2 years after the diagnosis. ${ }^{43}$ Additionally, the relationship between PNES frequency and health-related quality of life is influenced by psychopathological severity and other physical symptoms, ${ }^{44}$ bringing to light the importance of these other measures that are usually considered "secondary."

Table 1 summarizes the factors enumerated here that have implications in the development and conduction of PNES clinical trials.

\section{Presentation of the diagnosis: the first therapeutic intervention}

After the diagnosis is confirmed, presenting the diagnosis to the patient and, when appropriate, to his or her family, becomes the first therapeutic step toward recovery. Marked reduction in health care demand after diagnosis presentation has been established by prospective service evaluations. ${ }^{45,46}$ For a subset of PNES individuals, awareness that they do not suffer from epilepsy and that their events have emotional

Table I Limitations in the conduction of psychogenic nonepileptic seizures (PNES) clinical trials $s^{9,14,40-42}$

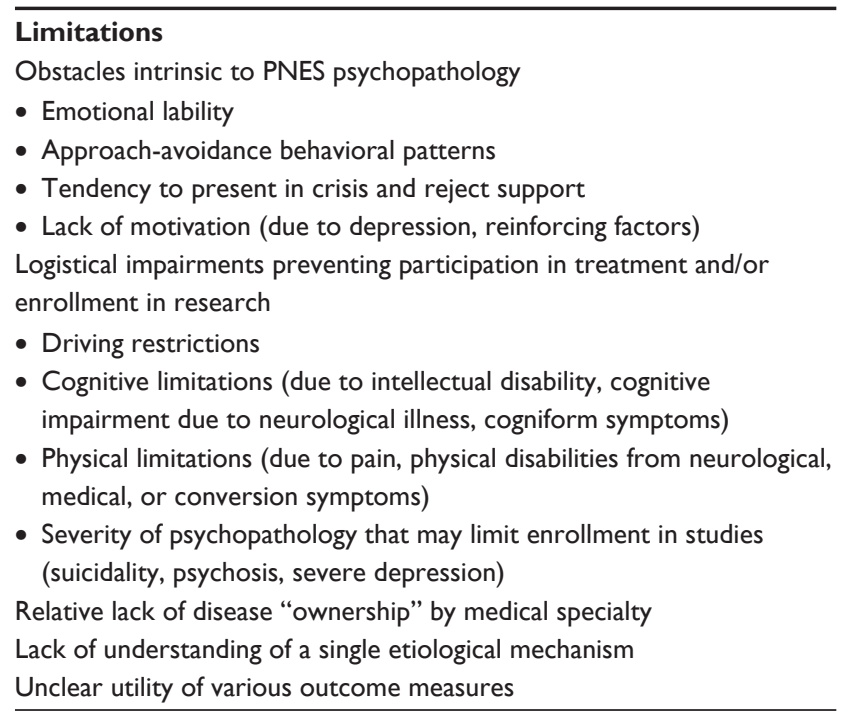

underpinnings is sufficient for the episodes to be under control, at least for the subsequent 6 months. ${ }^{47}$ Kanner et al ${ }^{48}$ described this subgroup of patients with sustained control of their attacks as having a lower degree of psychopathology and the ability to recognize stressful events as precipitating factors for their attacks.

How the PNES diagnosis is presented has always been considered a delicate matter, and protocols on how to present the diagnosis have been suggested. ${ }^{41,49}$ Although immediate relief after diagnosis presentation has been documented, ${ }^{50,51}$ maintenance of such immediate improvement has been variable. ${ }^{48,52}$ Because engagement in treatment will be crucial for recovery, and because PNES subjects are known to have low rates of treatment retention, ${ }^{53,54}$ this "first therapeutic step" is a decisive moment that may define the patient's clinical and functional outcome and hence it should be handled carefully.

A standardized protocol for diagnosis presentation was used in 50 newly diagnosed PNES subjects in a multicenter study. The protocol covered 14 points addressing all domains of patients' illness representations and it was found to be acceptable to patients. Fourteen percent at 2 weeks after the diagnosis and $20 \%$ at 11 weeks after the diagnosis reported attack freedom or only occasional events. ${ }^{55}$

Not uncommonly, health professionals may misinterpret the psychogenic origin of these events as a sign of voluntary fabrication. Understanding PNES as "faked seizures" may generate a negative attitude toward patients, and it can behaviorally reinforce reluctance in the patient to accept the diagnosis. Although there is no neurobiological evidence for PNES at this juncture, it is known from functional neuroimaging studies in psychogenic tremor and psychogenic paralysis $^{56-58}$ that brain activation differs between feigned and psychogenic symptoms, lending support to the "brain basis" of the disorder.

Table 2 summarizes the key points for a successful presentation of the diagnosis based on previous recommendations $\mathrm{s}^{49,55}$ and the author's own clinical experience at a nonepileptic seizure intervention clinic previously held at the University of Illinois Medical Center at Chicago.

\section{PNES: renewed interest?}

The first decade of the new millennium has seen an increase in interest in PNES. A limited search of the PubMed database for articles utilizing terms related to PNES in the title or abstract showed there were 96 articles published on the topic during the 1980s, 243 articles published during the 1990s, and 426 articles published during the 2000s. This growing interest in the topic has propelled a renewed enthusiasm in 
Table 2 Psychogenic nonepileptic seizures (PNES): how should the diagnosis be presented?

\begin{tabular}{|c|c|}
\hline Presentation & Description \\
\hline $\begin{array}{l}\text { Multidisciplinary } \\
\text { presentation }\end{array}$ & $\begin{array}{l}\text { The neurologist making the diagnosis and the } \\
\text { mental health professional who will follow the } \\
\text { patient are physically present when the diagnosis } \\
\text { is revealed and discussed, and they both agree } \\
\text { on the treatment plan }\end{array}$ \\
\hline $\begin{array}{l}\text { Objective discussion } \\
\text { of findings }\end{array}$ & $\begin{array}{l}\text { Description of the event as observed during } \\
\text { video recording and the lack of ictal discharges, } \\
\text { ruling out an epileptic etiology }\end{array}$ \\
\hline $\begin{array}{l}\text { Providers believe } \\
\text { the diagnosis }\end{array}$ & $\begin{array}{l}\text { Emphasis that the attacks are still considered } \\
\text { real and out of the patient's control }\end{array}$ \\
\hline $\begin{array}{l}\text { Explanation of the } \\
\text { "psychogenic" nature } \\
\text { of the attacks }\end{array}$ & $\begin{array}{l}\text { An individualized hypothetical explanation is given } \\
\text { on how these episodes may be taking place } \\
\text { Examples of trait vulnerabilities are highlighted } \\
\text { Patients may also be presented with examples of } \\
\text { different forms of psychopathology (especially one } \\
\text { they do not suffer from) to help them understand } \\
\text { that other signs and symptoms in psychiatric } \\
\text { disorders may be perceived as involuntary as well } \\
\text { The explanation may include discussion about } \\
\text { the patient's "brain" managing certain stressful } \\
\text { situations in a particular manner }\end{array}$ \\
\hline $\begin{array}{l}\text { Psychotherapy } \\
\text { referral }\end{array}$ & $\begin{array}{l}\text { Psychotherapeutic interventions are introduced } \\
\text { as an opportunity to learn new ways of relating } \\
\text { to physical and emotional experiences, reducing } \\
\text { vulnerability toward PNES, not as a promise to } \\
\text { eradicate PNES for life } \\
\text { When appropriate, redefinition of family } \\
\text { involvement is discussed }\end{array}$ \\
\hline $\begin{array}{l}\text { Psychiatric } \\
\text { comorbidities }\end{array}$ & $\begin{array}{l}\text { Emphasis is placed on the treatment of psychiatric } \\
\text { comorbidities with both psychopharmacological } \\
\text { and psychotherapeutic interventions }\end{array}$ \\
\hline $\begin{array}{l}\text { Involvement of } \\
\text { neurologist post } \\
\text { diagnosis }\end{array}$ & $\begin{array}{l}\text { Neurologists should remain involved and } \\
\text { available and work collaboratively with mental } \\
\text { health professionals to facilitate antiepileptic drug } \\
\text { withdrawal, treatment of comorbid neurological } \\
\text { conditions (including epilepsy), ongoing } \\
\text { evaluation should new events or symptoms arise, } \\
\text { and overall monitoring of the patient's outcome }\end{array}$ \\
\hline
\end{tabular}

identifying treatment interventions for this population. The following section will review treatment studies that have been published since the year 2000 .

\section{Treatment review Methods}

A PubMed literature search was conducted, focusing on treatment studies on PNES from January 2000 through December 2011. The search included articles with the terms "nonepileptic seizures" (as well as "non-epileptic seizures"), "psychogenic seizures," "dissociative seizures," "conversion seizures," "pseudoseizures," "psychogenic spells," and "psychogenic attacks" in the title or abstract. The results of these searches were combined with the Medical Subject Headings term "therapeutics." Titles and abstracts were reviewed to identify potentially relevant articles, which were then retrieved to review the full article. All results were limited to Englishlanguage articles and a study was included if it recruited all adult PNES patients only. The intervention being investigated had to be described as being delivered as uniformly as possible to all subjects. Case reports and case series were excluded. The main aspects of these studies are summarized in Table 3.

\section{Results: psychotherapeutic interventions CBT}

CBT is based on the concept that dysfunctional conditioned responses and thought processes lead to a misperception of reality that presents as psychopathological symptoms. ${ }^{14}$ In the case of CBT, PNES are conceptualized as dissociative responses to arousal when a patient is confronted with stimuli or circumstances that the patient tends to avoid, either consciously or not. ${ }^{59}$

CBT is the only psychotherapeutic intervention studied in PNES in a pilot randomized, controlled trial ${ }^{60}$ and it is therefore the psychotherapeutic treatment with the highest level of efficacy evidence (Class III) in this population. The content of Goldstein et al's ${ }^{59,60} \mathrm{CBT}$ approach emphasizes the following concepts in different stages through twelve outpatient sessions: (1) engagement in treatment; (2) reinforcement of independence; (3) distraction, relaxation, and refocusing techniques at the earliest signs of an event; (4) graded exposure to avoided situations; (5) cognitive restructuring; and (6) relapse prevention. In Goldstein et al's ${ }^{60}$ randomized, controlled trial, both the active and control groups received "standard medical care" (SMC) treatment, comprising up to seven neuropsychiatric appointments that focused on psychoeducation, support measures, and antiepileptic drug withdrawal. Event frequency was not different between analyzed groups (CBT + SMC, $\mathrm{n}=33$; SMC [control], $\mathrm{n}=31)$ at the start of treatment (CBT + SMC group median, twelve events per month; SMC group median, eight events per month), but significantly lower frequency for the $\mathrm{CBT}$ group at the end of the 12-session treatment (SMC group median, 6.75 events per month; CBT + SMC group median, two events per month) $(P=0.002)$, with a large between-group effect size $(0.75)$. At the 6-month follow-up, the between-group effect size (SMC group median, five events per month; CBT + SMC group median, 1.5 events per month) was medium (0.42) and not statistically significant $(P=0.082)$. Therapist contact was greater in the active group, and this level of contact was not controlled for in the control group, which may explain the 
Table 3 Treatment trials conducted in psychogenic nonepileptic seizures (PNES)

\begin{tabular}{|c|c|c|c|c|}
\hline \multirow[t]{2}{*}{ Reference } & \multicolumn{4}{|l|}{ Methods } \\
\hline & Trial design & Intervention & Final analysis (n) & Dependent variables \\
\hline Goldstein et $\mathrm{al}^{60}$ & Randomized, controlled & $\begin{array}{l}\text { CBT arm: I } 2 \text { weekly or } \\
\text { fortnightly hour-long sessions } \\
\text { SMC (both arms): supportive } \\
\text { sessions with psychoeducation } \\
\text { and AED withdrawal }\end{array}$ & 64 & $\begin{array}{l}\text { Monthly event frequency, rate of } \\
\text { event freedom, Work and Social } \\
\text { Adjustment Scale, Hospital Anxiety } \\
\text { and Depression Scale, modified } \\
\text { Client Service Receipt Inventory }\end{array}$ \\
\hline LaFrance et $\mathrm{al}^{61}$ & $\begin{array}{l}\text { Open-label, prospective, } \\
\text { uncontrolled }\end{array}$ & $\begin{array}{l}\text { I } 2 \text { weekly hour-long } \\
\text { CBT sessions }\end{array}$ & 20 & $\begin{array}{l}\text { Weekly event frequency, BDI, } \\
\text { Modified HDRS, Davidson Trauma } \\
\text { Scale, DES, BIS, Family Assessment } \\
\text { Device, SCL-90, Oxford Handicapped } \\
\text { Scale, Ways of Coping, QOLIE-3I }\end{array}$ \\
\hline Kuyk et al ${ }^{62}$ & $\begin{array}{l}\text { Open-label, prospective, } \\
\text { uncontrolled }\end{array}$ & $\begin{array}{l}\text { Inpatient individualized } \\
\text { CBT-based treatment for } \\
2-6 \text { months (average, } \\
4.8 \text { months) }\end{array}$ & 22 & $\begin{array}{l}\text { Average weekly event frequency, } \\
\text { SCL-90, BDI, State-Trait Inventory, } \\
\text { Utrecht Coping List, SF-36, } \\
\text { Dissociation Questionnaire }\end{array}$ \\
\hline
\end{tabular}

\begin{tabular}{|c|c|c|c|c|}
\hline Mayor et $\mathrm{al}^{64}$ & $\begin{array}{l}\text { Retrospective, } \\
\text { naturalistic study, } \\
\text { uncontrolled }\end{array}$ & $\begin{array}{l}\text { Up to } 20 \text { sessions of brief } \\
\text { augmented psychodynamic } \\
\text { interpersonal therapy }\end{array}$ & 47 & $\begin{array}{l}\text { Monthly event frequency, } \\
\text { SF-36, PHQ-I5 }\end{array}$ \\
\hline Barry et al ${ }^{66}$ & $\begin{array}{l}\text { Open-label, prospective, } \\
\text { uncontrolled }\end{array}$ & $\begin{array}{l}32 \text { weekly } 90 \text {-minute group } \\
\text { psychodynamic } \\
\text { psychotherapy sessions }\end{array}$ & 7 & $\begin{array}{l}\text { Weekly event frequency, } \\
\text { BDI, SCL-90 }\end{array}$ \\
\hline Prigatano et al $^{67}$ & $\begin{array}{l}\text { Open-label, prospective, } \\
\text { uncontrolled }\end{array}$ & $\begin{array}{l}24 \text { weekly } 90 \text {-minute } \\
\text { group psychoeducational } \\
\text { interventions }\end{array}$ & 9 & $\begin{array}{l}\text { Weekly event frequency, MMPI-2, } \\
\text { and neurocognitive measures } \\
\text { (WAIS-III, CVLT, or RAVLT) }\end{array}$ \\
\hline Zaroff et al ${ }^{68}$ & $\begin{array}{l}\text { Open-label, prospective, } \\
\text { uncontrolled }\end{array}$ & $\begin{array}{l}\text { I0 weekly hour-long } \\
\text { group psychoeducational } \\
\text { interventions }\end{array}$ & 7 & $\begin{array}{l}\text { Pre- and posttreatment event } \\
\text { frequency, Coping Inventory } \\
\text { for Stressful Situations, Davidson } \\
\text { Trauma Scale, Curious Experiences } \\
\text { Survey, STAXI-2, QOLIE-3I }\end{array}$ \\
\hline
\end{tabular}




\begin{tabular}{|c|c|}
\hline & Results \\
\hline \multicolumn{2}{|l|}{ Data collection method } \\
\hline $\begin{array}{l}\text { Data collected at start of treatment, end } \\
\text { of treatment, and at 6-month follow-up }\end{array}$ & $\begin{array}{l}\text { Significantly lower event frequency at treatment } \\
\text { end and a trend for lower event frequency } \\
\text { at 6-month follow-up following CBT } \\
\text { Large between-group effect size at end of } \\
\text { treatment and medium effect size at follow-up } \\
\text { No difference between groups in secondary } \\
\text { measures }\end{array}$ \\
\hline
\end{tabular}

Event frequency obtained for the week prior to enrollment, after enrollment, weekly during treatment, and at completion Measures were obtained at baseline, during treatment, and after treatment Average weekly event frequency for previous 3 weeks at onset and end of treatment; average weekly event frequency for previous 4 weeks at 6-month follow-up

Secondary measures also obtained at onset of treatment, discharge, and 6-month follow-up

Questionnaire data collected 50 months (median) after baseline measures and 42 months (median) after end of treatment

Psychosocial functioning measures obtained at baseline

Data collected at start of treatment, weekly for event frequency, and at 16 and 32 weeks for secondary measures

Weekly event log; weekly quiz about previous session MMPI-2 and neurocognitive evaluation obtained during assessment period

Pre- and posttreatment administration of all measures

Pretreatment questionnaire scores were compared with normative data (except event frequency) and with posttreatment score

Mean event frequency decreased significantly from week I through end of treatment Most scales assessing secondary outcome measures showed significant improvement from baseline to final session (except HDRS and DES) Significant decrease in event frequency from onset to discharge, from discharge to follow-up, and from onset to follow-up

$27.3 \%$ achieved event freedom at end of treatment and $44 \%$ at follow-up Significant decrease in BDI, State-Trait Inventory, dissociation questionnaire, and Utrecht Coping List from onset to discharge and in all measures from onset to follow-up

At follow-up (median, 42 months after end of treatment), $25.5 \%$ of patients had become event free; a further $40.4 \%$ achieved an event reduction of $>50 \%$

Health care utilization declined significantly from baseline to follow-up, based on questionnaire

Six of seven patients with decrease in event frequency over course of treatment Four of seven with event cessation Five subjects remained event free several months after treatment Significant reduction in BDI over the course of treatment

Meaningful changes in ten of twelve SCL-90 subscales

Six patients reported a decrease in event frequency, two reported no change,

and one reported an increase Significant correlation between MMPI-2 paranoid scale and frequency of events (positive) and correct answers in quiz (negative)

Typical conversion $\mathrm{V}$ profile observed No change in event frequency in four subjects (three had achieved remission at treatment initiation), a decrease in two subjects, and an increase in one subject Significant reduction in Curious Experience Survey, Davidson Trauma Scale, and Coping Inventory for

\section{Comments}

SMC group not controlled for therapist contact (which was greater in CBT) Nature of treatment precludes blinding from SMC provider

Selection bias in favor of chronic, more difficult-to-treat patients

Willingness to enroll in study might have influenced favorable outcome in SMC group 6-month follow-up period too short for change in employment status Only individuals with v-EEG diagnosed PNES were included Stressful Situations (Emotion Subscale), and STAXI-2 Anger Expression Index Score Increase in QOLIE-3I
Article states follow-up questionnaires were administered at months 4,8 , and 12 from the date of enrollment (although data not available) Diagnosis confirmed via EEG; comorbid epilepsy subjects excluded from analysis Inpatient setting not controlled for Treatment was individualized, limiting generalizability of results Sample selection bias toward patients with less severe psychiatric comorbidities, as this was an exclusion criterion for participation

Some patients did not obtain PNES diagnosis with v-EEG

Contact with other providers and antidepressant treatment may have influenced outcomes No predefined "time point" after therapy Measurements of social functioning only obtained at baseline, not at follow-up Five patients receiving individual psychotherapy concurrently, one with new antidepressant

Two series of six and seven patients each; first series with no exclusion criteria; second series required pretreatment interview One patient had comorbid epilepsy

All had v-EEG-confirmed diagnosis Three of seven subjects had event freedom at treatment initiation 
Table 3 (Continued)

\begin{tabular}{|c|c|c|c|c|}
\hline \multirow[t]{2}{*}{ Reference } & \multicolumn{4}{|l|}{ Methods } \\
\hline & Trial design & Intervention & Final analysis (n) & Dependent variables \\
\hline LaFrance et al ${ }^{69}$ & $\begin{array}{l}\text { Randomized, } \\
\text { double-blind, } \\
\text { placebo-controlled }\end{array}$ & $\begin{array}{l}\text { Sertraline } 25-200 \mathrm{mg} \text { daily } \\
\text { versus placebo }\end{array}$ & 33 & $\begin{array}{l}\text { Fortnightly event frequency, BDI, } \\
\text { Modified HDRS, Davidson Trauma } \\
\text { Scale, DES, BIS, Family Assessment } \\
\text { Device, SCL-90, GAF, Oxford } \\
\text { Handicapped Scale, Ways of } \\
\text { Coping, QOLIE-3 I }\end{array}$ \\
\hline
\end{tabular}

$\begin{array}{llll}\text { Pintor et } \mathrm{al}^{70} & \begin{array}{l}\text { Open-label, prospective, } \\ \text { uncontrolled }\end{array} & \begin{array}{l}\text { Venlafaxine } 75-300 \mathrm{mg} \text { daily } \\ \text { by clinician criteria }\end{array} & \begin{array}{l}\text { Monthly event frequency, HDRS, } \\ \text { All subjects with } \\ \text { depressive and/or } \\ \text { anxiety disorder }\end{array}\end{array}$

\begin{abstract}
Abbreviations: AED, antiepileptic drug; BDI, Beck Depression Inventory; BIS, Barrett Impulsivity Scale; CBT, cognitive behavioral therapy; CVLT, California Verbal Learning Test; DES, Dissociative Experiences Scale; EEG, electroencephalography; GAF, Global Assessment of Functioning; HARS, Hamilton Anxiety Rating Scale; HDRS, Hamilton Depression Rating Scale; MMPI-2, Minnesota Multiphasic Personality Inventory 2; PHQ-I5, Patient Health Questionnaire I5-Item Somatic Symptom Severity Scale; QOLIE-3I, Quality of Life in Epilepsy Inventory 3I; RAVLT, Rey Auditory Verbal Learning Test; SCID-I, Structured Clinical Interview for DSM-IV Axis I Disorders; SCL-90, Symptoms Checklist-90; SF-36, 36-Item Short Form Health Survey; SMC, standard medical care; STAXI-2, State-Trait Anger Expression Inventory-2; v-EEG, videoelectroencephalography; WAIS-III, Wechsler Adult Intelligence Scale-III.
\end{abstract}

decrease in between-group difference over time. At the same time, willingness to enroll in this study may have influenced the control group's reduction in events.

LaFrance et al's ${ }^{61}$ uncontrolled CBT study had 20 subjects enrolled and 17 completed a 12-week outpatient CBT protocol. The protocol was driven by similar principles as those of or Goldstein et al's study, ${ }^{60}$ but it specifically followed a manual that focused on several points including mood-cognitionenvironment connections; identification of moods, situations, and thoughts; relaxation techniques; healthy communication; and examination of internal and external triggers. Mean event frequency decreased significantly from week 1 (mean event frequency, 17.2 per week) through the end of treatment (mean event frequency, 7.1 per week) $(P=0.001)$. Eleven of the 17 subjects completing the study $(65 \%)$ reported no events per week during the final CBT session. Mean scores on measures of depression, anxiety, somatic symptoms, quality of life, and psychosocial functioning showed significant improvement from baseline to the end of treatment. The study does not contain follow-up data beyond week 12, motivating future trials to examine if these strong results can be maintained over time.

An uncontrolled, prospective inpatient treatment program based on CBT principles has also provided evidence of reduction of seizure-like events. ${ }^{62}$ The treatment was provided over a prolonged inpatient admission, that allowed home visits during weekends, with a 4-week psychological diagnostic process, followed by a multidisciplinary treatment aimed at cognitive restructuring, trauma treatment, stimulus differentiation, coping skills training, stress management, behavioral analysis, and rational emotive therapy, all provided in individual and/or group settings. Family interventions were also included. The duration of the program ranged from 2 to 6 months, with an average duration of 4.8 months. The 22 completers showed a significant reduction between treatment initiation (mean event frequency, 6.6 per week) and end of treatment (mean event frequency, 3.0 per week) $(P=0.02)$ and 16 of them showed a significant reduction between treatment initiation and the 6-month follow-up after discharge (mean event frequency, 0.9 per week $(P=0.002)$. Reductions between initiation of treatment and the 6-month follow-up period were also observed in measures of depression, anxiety, dissociation, health-related quality of life, and overall psychopathology. Six months after discharge, $80 \%$ of 16 patients had an event reduction of over $50 \%$ and half of these patients were event free. The prolonged inpatient setting where the treatment took place and the incorporation of several treatment strategies may have contributed to these positive results. At the same time, the multimodal nature of the treatment and the setting where it took place may represent limitations when it comes to adoption of this therapeutic approach for most PNES patients. Willingness to be admitted to an inpatient unit for over 2 months may represent a selection bias toward patients already open to the diagnosis.

\section{Psychodynamic psychotherapies: augmented psychodynamic interpersonal therapy and group psychodynamic psychotherapy}

According to psychodynamic theory, symptoms and behaviors are presumed to be influenced by internal processes, 


\begin{tabular}{|c|c|c|}
\hline & Results & Comments \\
\hline \multicolumn{3}{|l|}{ Data collection method } \\
\hline $\begin{array}{l}\text { Initial visit: SCID-I and pretreatment 2-week } \\
\text { event frequency and baseline measures }\end{array}$ & $\begin{array}{l}\text { No difference between treatment groups } \\
\text { regarding event frequency by risk ratios }\end{array}$ & $\begin{array}{l}\text { Not powered for establishing treatment } \\
\text { efficacy because of limited sample size }\end{array}$ \\
\hline Follow-up visit 2 weeks later with & Relative change in biweekly event frequency from & Study not stratified to differentiate based \\
\hline initiation of blinded treatment, biweekly & baseline to study end, separately, by treatment & on presence of personality disorders \\
\hline visits for 12 weeks with measurement & group with $45 \%$ decrease in the sertraline group & \\
\hline of event frequency & versus $8 \%$ increase in the placebo group & \\
\hline All measures and 2-week event & No difference between groups in secondary & \\
\hline frequency at week 12 & measures & \\
\hline Initial visit: SCID-I and washout for & Statistically significant reduction in all variables & Inclusion in study within I-2 months of \\
\hline 15 days, if necessary & (including event frequency and depression and & diagnosis (rather than a year) \\
\hline Follow-up visit 15 days later with initiation & anxiety scales) & No direct efficacy outcomes on conversion \\
\hline of venlafaxine treatment, monthly visits & No difference in patients with more than & symptoms in absence of affective or anxiety \\
\hline for 5 months with measurement with & ten events in 15 days & symptoms \\
\hline standardized scales (except SCID-I) & & No measurement of side effects \\
\hline
\end{tabular}

many times unconscious to the patient, with childhood experiences being highly influential to the development of persistent maladaptive behavioral patterns. ${ }^{14}$ Defense mechanisms such as dissociation and somatization are used to split traumatic memories from consciousness. ${ }^{63}$ The goal of psychodynamic therapy is to increase the patient's awareness of these patterns to facilitate change. ${ }^{14}$

Mayor et $\mathrm{l}^{64}$ studied brief augmented psychodynamic psychotherapy in 47 PNES subjects. ${ }^{64}$ Key features from this kind of psychotherapy include the assumption that the patient's problems are linked to difficulties in his or her personal relationships, which are explained by interpersonal patterns developed earlier in life. The therapeutic mechanism of this approach is based on the identification and change of unhelpful interpersonal patterns and the more effective processing of emotions related to both present and past issues. Techniques from somatic trauma therapy, such as control of autonomic arousal, tracking of somatic symptoms, linkage to emotional triggers, and processing of traumatic memories, are used to "augment" the psychodynamic interpersonal approach. ${ }^{65}$ The 47 subjects completed up to 20 weekly or fortnightly sessions (range, one to eighteen sessions; median, five sessions); twelve of these subjects (26\%) achieved event freedom 42 months (median) after the conclusion of therapy and a further 19 subjects (40\%) reported more than 50\% reduction in event frequency compared with baseline. The median frequency of events per month decreased from six at baseline to one at follow-up $(P<0.007)$. There was also a significant decline in health care utilization at follow-up.
Some of these patients had not obtained the diagnosis of PNES through v-EEG confirmation, relying on clinical impression by a neurologist with expertise in epilepsy, direct observation of the events in clinic, video recordings, or a history or witness account suggestive of PNES. Contact with other providers, such as a neurologist, or prescription of antidepressant or anxiolytic medication may have influenced improvement as well.

A few studies have evaluated group psychotherapy in the treatment of PNES. Barry et $\mathrm{al}^{66}$ reported on the efficacy of a 32-week-long psychodynamically oriented group psychotherapy, which had as a goal the conscious and verbal expression of emotional distress, obviating the need for somatic displays of stress. Development of assertive coping strategies instead of passive avoidant behavior was emphasized. Six of the seven $(86 \%)$ completers experienced a decrease in frequency of events over the treatment period. Four of the seven $(57 \%)$ achieved complete remission, with very occasional recurrence in the face of emotional distress. Depression severity and overall severity of psychopathology also showed improvement.

\section{Other group interventions}

Prigatano et al ${ }^{67}$ reported a decrease in six of nine subjects (67\%) with PNES during completion of a 6-month group psychotherapy program. The treatment consisted of 24 90-minute sessions that reviewed facts about epilepsy and PNES and where discussion of feelings that may relate to past or present events relevant to PNES was encouraged. 
Results were based on a comparison between number of events during the first twelve sessions and number of events during the second twelve sessions.

Zaroff et a $1^{68}$ reported on the efficacy of a 1-hour weekly group psychotherapy intervention over the course of 10 weeks. The approach was psychoeducational in nature and it covered topics including PNES, anger, trauma and abuse, somatization tendencies, quality of life, and stress management techniques, among others. Four of the seven completers (57\%) who were enrolled in this program experienced no change in event frequency, but in three of these four subjects ( $75 \%)$, event cessation had been achieved at treatment initiation. Two subjects experienced a decrease and one an increase in event frequency. Decreases were seen in post-traumatic and dissociative symptoms and emotionally based coping mechanisms. The report does not mention if patients also received additional individual psychotherapy or psychopharmacotherapy during the group intervention.

\section{Results: psychopharmacological interventions}

The lack of a consistent neurobiological model in PNES makes identification of a customized neurochemical intervention unrealistic at this stage. However, under the premise that difficulties with impulsivity, compulsive tendencies, depression, and anxiety are associated with serotonergic deficits and these conditions are usually seen comorbidly in PNES, ${ }^{14}$ serotonin reuptake inhibitors, both selective and dual action, have been investigated as potentially useful compounds.

LaFrance et $\mathrm{a}^{69}$ conducted a randomized, double-blind, placebo-controlled trial evaluating the efficacy of flexibledose sertraline over 12 weeks at reducing PNES frequency and improving other psychiatric severity and psychosocial measures. The final analysis included 33 subjects with one or more events in the 2 weeks prior to enrollment. Patients were allowed to participate if taking other antidepressants, except for monoamine oxidase inhibitors and sertraline at a $100 \mathrm{mg} /$ day or higher dose for more than 30 days. Dose of the concurrent antidepressants, if prescribed, was held constant. There was no difference between groups in fortnightly event frequency change (risk ratio, $0.51 ; P=0.29$ ). However, when groups were analyzed separately, the sertraline arm showed a $45 \%$ decrease in biweekly event frequency $(P=0.03)$, while the control group showed an $8 \%$ increase $(P=0.78)$. There were no between-arm differences in secondary outcome measure changes. The limited sample size means this wellconducted study is not powered enough to establish treatment efficacy. The sertraline group had a higher rate of baseline event frequency in those subjects with Axis II disorders than in those without Axis II disorders; this baseline difference was not observed in the control group. The study was not stratified to differentiate results based on the presence of personality disorders.

An open-label, prospective, uncontrolled study of flexible-dose venlafaxine evaluated event reduction as well as anxiety and depression severity. ${ }^{70}$ All enrolled subjects had v-EEG-confirmed PNES but also had to meet criteria for a unipolar depressive disorder and/or anxiety disorder. Subjects underwent monthly assessments for 5 months. The 19 subjects who completed the study showed a statistically significant reduction in all symptom scales and monthly event frequency at the fifth-month assessment compared with the initial assessment. There was no difference in patients with more than ten events in the 15-day baseline pre-inclusion assessment period. That the inclusion of PNES subjects after diagnosis was confirmed no earlier than 2 months from enrollment may have assisted in the attention provided to the diagnosis; however, this may be irrelevant, as the mean duration of PNES was 6 years in this study.

\section{Discussion and future directions}

One pilot psychopharmacotherapeutic study meeting criteria for Class II evidence ${ }^{69}$ and one pilot psychotherapeutic study meeting criteria for Class III evidence ${ }^{60}$ are the scientifically strongest evidence currently available for the treatment of PNES. ${ }^{71}$ Both studies had limited power, and while strong support cannot be claimed for these interventions at this point, it is appropriate to say that these are very promising approaches that require further study. This limited evidence shows how early the current stage is in the development of therapeutic interventions for this clinical entity. The lack of a clear-cut etiological model that supports the psychogenic origin of PNES, and the heterogeneous background that can lead to a similar phenotypic presentation, can make finding an explanation for the disorder and a one-size-fits-all intervention a difficult task to achieve. On the other hand, the field of psychiatry is known to accommodate etiological uncertainties and a wide variety of risk factors that can lead to a specific symptom display while still investing in the development of treatment interventions. Why are PNES lagging behind then in treatment development? Is the fact that many of these patients are initially thought to have epilepsy a reason for this cold embrace and, to some degree, skepticism? 
Because most PNES patients enter diagnosis and treatment through the "epilepsy" door, the collaboration between neurology and psychiatry is of utmost relevance. Dual training or neuropsychiatric specialization may help bridge this dichotomy, although there may be a limited number of professionals with such background and interest. "Treatment as usual" for PNES mostly depends on a referral to a mental health professional. ${ }^{10}$ For individuals who accept the diagnosis without hesitation, who do not possess chronic risk factors that perpetuate their symptoms, and who are able to find a competent professional comfortable treating PNES, "treatment as usual" may prove therapeutic. However, for many patients, limited acceptance of the diagnosis, a number of chronic psychiatric difficulties, and limited therapeutic resources still represent barriers to adequate treatment that can lead to a successful outcome.

A multidisciplinary and flexible model for the treatment of PNES may provide the background infrastructure necessary upon which the specific psychiatric interventions may be deployed successfully. Beyond the development of this infrastructure, understanding the vulnerabilities that lead to PNES may help customize many of the interventions with the goal of providing long-term beneficial outcomes. While the understanding of these vulnerability traits is still at an early stage,${ }^{8}$ these traits may be key ingredients that need to be recognized and addressed for a durable, positive outcome in therapeutic trials and actual practice. Additionally, the role of the therapeutic relationship cannot be underestimated, as it may provide the basis for further adaptive development of emotional expression and identity. ${ }^{72}$

Although a case series and therefore not included in the author's earlier analysis, Rusch et $\mathrm{al}^{11}$ reported on 26 PNES adults who received diverse psychotherapeutic interventions of varying duration based on the symptom pattern underlying their PNES presentation. All subjects except one experienced either cessation or improvement of their events. Treatment was not delivered in a standardized fashion. This article is mentioned because, although not a standardized intervention, it does capture the essence of the heterogeneous patient population that PNES patients are, and it demonstrates how, even with individualized treatment, follow-up data can be prospectively tracked.

Evaluation of structured therapies such as CBT, psychodynamic therapies and group interventions, and serotonergic antidepressants in larger, randomized, controlled trials are necessary to establish solid guidelines for treatment. Event induction through hypnosis has been evaluated to help discriminate between epileptic seizures and PNES, ${ }^{73,74}$ creating interest in hypnotherapy as a potentially effective intervention. Hypnotherapy was studied in conversion, but not in PNES-only samples in a rigorous manner, limiting comparison with the interventions mentioned earlier. ${ }^{22,23}$ Additionally, other psychotherapeutic approaches such as mindfulness-based interventions, EMDR, and sensorimotor therapy have not been systematically studied and may be well suited for PNES.

Mindfulness-based interventions are rooted in the concept of mindfulness, "paying attention in a particular way: on purpose, in the present moment and non-judgmentally." $" 5$ Mindfulness has been articulated in specific psychotherapeutic approaches, such as acceptance and commitment therapy ${ }^{76}$ dialectical behavioral therapy ${ }^{77}$ mindfulness-based stress reduction, ${ }^{75}$ and mindfulness-based cognitive therapy. ${ }^{78}$ The application of mindfulness practice can lead to a shift in perspective, termed "reperceiving," which becomes an overarching mechanism of action under which more direct mechanisms, can lead to change and positive outcomes. ${ }^{79}$ The emphasis of mindfulness-based interventions on intentional and nonjudgmental attention in the present moment, followed by an acceptance stance and behavioral choice based on high-value roles, could help conversion and dissociation patients develop new strategies to eventually replace their maladaptive ones dictated by their vulnerability traits. ${ }^{80}$

Sensorimotor therapy has been specifically developed for the treatment of trauma and dissociation. The goal of sensorimotor therapy is to help the individual process his or her traumatic experience within a state of "optimal arousal," instead of the usually displayed hypearoused (ie, reexperiencing, hypervigilance) or hypoaroused (ie, dissociation, freezing response) states. ${ }^{81}$ Given the high prevalence of traumatic experiences and also the identified hypervigilance ${ }^{82}$ and avoidance tendencies in PNES subjects, ${ }^{83}$ this kind of treatment may hold some promise for at least a subset of patients.

EMDR has shown results comparable with those of CBT in the treatment of PTSD, and its proposed mechanism of action involves processing of trauma memories with new, positive information, separating the arousal associated with these memories. This is achieved by creating a vivid image of the traumatic memory while the patient is led through eye movements and other tactile stimulations. ${ }^{36}$ The creation of a new mechanism to process these memories without the associated arousal may also be fitting to PNES patients, especially considering the high prevalence of PTSD in this 
population. Although no rigorous studies were conducted in PNES, a case series of three subjects with PNES and PTSD rendered event remission, with this event remission being sustained for 12-18 months. ${ }^{84}$

Similarly, as more is learned about the underlying neurocircuitry dysfunction in PNES ${ }^{85}$ effective treatments may be strengthened by neuromodulatory interventions.

\section{Conclusion}

This review presented the current state in the treatment of PNES, including limitations and difficulties in conducting clinical trials in this population and a proposed model for diagnosis presentation. A review of the most recent medical literature was provided with a detailed description of those studies with the scientifically strongest evidence of efficacy. Finally, concerns regarding the delay in PNES treatment development and some practical ideas on how to create an infrastructure for successful treatment outcomes were discussed.

CBT and the antidepressant sertraline were evaluated in pilot double-blind, randomized, controlled trials. Other interventions evaluated in uncontrolled trials include augmented psychodynamic interpersonal psychotherapy, group psychodynamic psychotherapy, group psychoeducation and the antidepressant venlafaxine. These ineterventions all seem promising, but require further investigation in larger samples and/or with a more rigorous methodology. Successful outcomes in clinical practice will be dependent on adaptation and flexible delivery of these interventions.

\section{Disclosure}

The author reports no conflicts of interest in this work.

\section{References}

1. Reuber M, Fernández G, Bauer J, Helmstaedter C, Elger CE. Diagnostic delay in psychogenic nonepileptic seizures. Neurology. 2002;58(3): 493-495.

2. Benbadis SR, O’Neill E, Tatum WO, Heriaud L. Outcome of prolonged video-EEG monitoring at a typical referral epilepsy center. Epilepsia. 2004;45(9):1150-1153.

3. Martin RC, Gilliam FG, Kilgore M, Faught E, Kuzniecky R. Improved health care resource utilization following video-EEG-confirmed diagnosis of non-epileptic psychogenic seizures. Seizure. 1998;7(5): 385-390.

4. Szaflarski JP, Szaflarski M, Hughes C, Ficker DM, Cahill WT, Privitera MD. Psychopathology and quality of life: psychogenic non-epileptic seizures versus epilepsy. Med Sci Monit. 2003;9(4): CR113-CR118.

5. Testa SM, Schefft BK, Szaflarski JP, Yeh HS, Privitera MD. Mood, personality, and health-related quality of life in epileptic and psychogenic seizure disorders. Epilepsia. 2007;48(5):973-982.

6. Duncan R, Oto M, Wainman-Lefley J. Mortality in a cohort of patients with psychogenic non-epileptic seizures. J Neurol Neurosurg Psychiatry. 2012;83(7):761-762.
7. Reuber M. The etiology of psychogenic non-epileptic seizures: toward a biopsychosocial model. Neurol Clin. 2009;27(4):909-924.

8. Baslet G. Psychogenic non-epileptic seizures: a model of their pathogenic mechanism. Seizure. 2011;20(1):1-13.

9. LaFrance WC Jr, Alper K, Babcock D, et al; NES Treatment Workshop participants. Nonepileptic seizures treatment workshop summary. Epilepsy Behav. 2006;8(3):451-461.

10. LaFrance WC Jr, Rusch MD, Machan JT. What is "treatment as usual" for nonepileptic seizures? Epilepsy Behav. 2008;12(3):388-394.

11. Rusch MD, Morris GL, Allen L, Lathrop L. Psychological treatment of nonepileptic events. Epilepsy Behav. 2001;2(3):277-283.

12. Baslet G, Roiko A, Prensky E. Heterogeneity in psychogenic nonepileptic seizures: understanding the role of psychiatric and neurological factors. Epilepsy Behav. 2010;17(2):236-241.

13. D'Alessio L, Giagante B, Oddo S, et al. Psychiatric disorders in patients with psychogenic non-epileptic seizures, with and without comorbid epilepsy. Seizure. 2006;15(5):333-339.

14. LaFrance WC Jr, Barry JJ. Update on treatments of psychological nonepileptic seizures. Epilepsy Behav. 2005;7(3):364-374.

15. Brooks JL, Goodfellow L, Bodde NM, Aldenkamp A, Baker GA. Nondrug treatments for psychogenic nonepileptic seizures: what's the evidence? Epilepsy Behav. 2007;11(3):367-377.

16. Jongsma MJ, Mommers JM, Renier WO, Meinardi H. Follow-up of psychogenic, non-epileptic seizures: a pilot study; experience in a Dutch special centre for epilepsy. Seizure. 1999;8(3):146-148.

17. Aboukasm A, Mahr G, Gahry BR, Thomas A, Barkley GL. Retrospective analysis of the effects of psychotherapeutic interventions on outcomes of psychogenic nonepileptic seizures. Epilepsia. 1998;39(5):470-473.

18. Conder RL, Zasler ND. Psychogenic seizures in brain injury: diagnosis, treatment and case study. Brain Inj. 1990;4(4):391-397.

19. Nash JL. Pseudoseizures: etiologic and psychotherapeutic considerations. South Med J. 1993;86(11):1248-1252.

20. Stone J, Sharpe M, Binzer M. Motor conversion symptoms and pseudoseizures: a comparison of clinical characteristics. Psychosomatics. 2004;45(6):492-499.

21. Bowman ES, Markand ON. Psychodynamics and psychiatric diagnoses of pseudoseizure subjects. Am J Psychiatry. 1996;153(1):57-63.

22. Moene FC, Spinhoven P, Hoogduin KA, van Dyck R. A randomized controlled clinical trial of a hypnosis-based treatment for patients with conversion disorder, motor type. Int J Clin Exp Hypn. 2003;51(1): 29-50.

23. Moene FC, Spinhoven P, Hoogduin KA, van Dyck R. A randomised controlled clinical trial on the additional effect of hypnosis in a comprehensive treatment programme for in-patients with conversion disorder of the motor type. Psychother Psychosom. 2002;71(2): 66-76.

24. Dallocchio C, Arbasino C, Klersy C, Marchioni E. The effects of physical activity on psychogenic movement disorders. Mov Disord. 2010;25(4):421-425.

25. Hinson VK, Weinstein S, Bernard B, Leurgans SE, Goetz CG. Single-blind clinical trial of psychotherapy for treatment of psychogenic movement disorders. Parkinsonism Relat Disord. 2006;12(3):177-180.

26. Voon V, Lang AE. Antidepressant treatment outcomes of psychogenic movement disorder. J Clin Psychiatry. 2005;66(12):1529-1534.

27. Chastan N, Parain D. Psychogenic paralysis and recovery after motor cortex transcranial magnetic stimulation. Mov Disord. 2010;25(10): 1501-1504.

28. Kroenke K, Swindle R. Cognitive-behavioral therapy for somatization and symptom syndromes: a critical review of controlled clinical trials. Psychother Psychosom. 2000;69(4):205-215.

29. Guthrie E, Creed F, Dawson D, Tomenson B. A controlled trial of psychological treatment for the irritable bowel syndrome. Gastroenterology. $1991 ; 100(2): 450-457$.

30. Creed F, Fernandes L, Guthrie E, et al; North of England IBS Research Group. The cost-effectiveness of psychotherapy and paroxetine for severe irritable bowel syndrome. Gastroenterology. 2003;124(2): 303-317. 
31. O’Malley PG, Jackson JL, Santoro J, Tomkins G, Balden E, Kroenke K. Antidepressant therapy for unexplained symptoms and symptom syndromes. J Fam Pract. 1999;48(12):980-990.

32. Brand BL, Classen CC, McNary SW, Zaveri P. A review of dissociative disorders treatment studies. J Nerv Ment Dis. 2009;197(9):646-654.

33. Kellett $\mathrm{S}$. The treatment of dissociative identity disorder with cognitive analytic therapy: experimental evidence of sudden gains. J Trauma Dissociation. 2005;6(3):55-81.

34. Otte C. Cognitive behavioral therapy in anxiety disorders: current state of the evidence. Dialogues Clin Neurosci. 2011;13(4):413-421.

35. Stoffers JM, Völlm BA, Rücker G, Timmer A, Huband N, Lieb K. Psychological therapies for people with borderline personality disorder. Cochrane Database Syst Rev. 2012;8:CD005652.

36. Cukor J, Olden M, Lee F, Difede J. Evidence-based treatments for PTSD, new directions, and special challenges. Ann NYAcad Sci. 2010; 1208:82-89.

37. Ursano RJ, Bell C, Eth S, et al; Work Group on ASD and PTSD; Steering Committee on Practice Guidelines. Practice guideline for the treatment of patients with acute stress disorder and posttraumatic stress disorder Am J Psychiatry. 2004;161(Suppl 11):3-31.

38. American Psychiatric Association. Practice guideline for the treatment of patients with borderline personality disorder. Am J Psychiatry. 2001;158(Suppl 10):1-52.

39. Nijdam MJ, Gersons BP, Reitsma JB, de Jongh A, Olff M. Brief eclectic psychotherapy v. eye movement desensitisation and reprocessing therapy for post-traumatic stress disorder: randomised controlled trial. Br J Psychiatry. 2012;200(3):224-231.

40. LaFrance WC Jr. Conducting treatment trials for psychologic nonepileptic seizures. In: Schachter SC, Holmes GL, Kasteleijn-Nolst Trenité DGA, editors. Behavioral Aspects of Epilepsy: Principles and Practice. New York: Demos; 2008:421-430.

41. LaFrance WC Jr, Kanner AM, Barry JJ. Treating patients with psychological nonepileptic seizures. In: Ettinger AB, Kanner AM, editors. Psychiatric Issues in Epilepsy. 2nd ed. Philadelphia: Lippincott Williams \& Wilkins; 2007:461-488.

42. Chalder T. Cognitive behavioural therapy as a treatment for conversion disorder. In: Halligan PW, Bass C, Marshall JC, editors. Contemporary Approaches to the Study of Hysteria. New York: Oxford University Press; 2001:298-311.

43. Reuber M, Mitchell AJ, Howlett S, Elger CE. Measuring outcome in psychogenic nonepileptic seizures: how relevant is seizure remission? Epilepsia. 2005;46(11):1788-1795.

44. Lawton G, Mayor RJ, Howlett S, Reuber M. Psychogenic nonepileptic seizures and health-related quality of life: the relationship with psychological distress and other physical symptoms. Epilepsy Behav. 2009; 14(1):167-171.

45. Jirsch JD, Ahmed SN, Maximova K, Gross DW. Recognition of psychogenic nonepileptic seizures diminishes acute care utilization. Epilepsy Behav. 2011;22(2):304-307.

46. Razvi S, Mulhern S, Duncan R. Newly diagnosed psychogenic nonepileptic seizures: health care demand prior to and following diagnosis at a first seizure clinic. Epilepsy Behav. 2012;23(1):7-9.

47. Duncan R, Razvi S, Mulhern S. Newly presenting psychogenic nonepileptic seizures: incidence, population characteristics, and early outcome from a prospective audit of a first seizure clinic. Epilepsy Behav. 2011;20(2):308-311.

48. Kanner AM, Parra J, Frey M, Stebbins G, Pierre-Louis S, Iriarte J. Psychiatric and neurologic predictors of psychogenic pseudoseizure outcome. Neurology. 1999;53(5):933-938.

49. Shen W, Bowman ES, Markand ON. Presenting the diagnosis of pseudoseizure. Neurology. 1990;40(5):756-759.

50. Buchanan N, Snars J. Pseudoseizures (non epileptic attack disorder): clinical management and outcome in 50 patients. Seizure. 1993;2(2): 141-146.

51. Farias ST, Thieman C, Alsaadi TM. Psychogenic nonepileptic seizures: acute change in event frequency after presentation of the diagnosis. Epilepsy Behav. 2003;4(4):424-429.
52. Wilde C, Marquez AV, Farias ST, et al. Long-term follow-up study of patients with PNES. Epilepsia. 2004;45(Suppl 7):S349.

53. Baslet G, Prensky E, Roiko A, Uliaszek A, Prasad P. Initial Treatment Retention in Psychogenic Nonepileptic Seizures. In: Abstracts from the 22nd American Neuropsychiatric Association Annual Meeting; 2011 March 23-26; Denver, USA. Journal of Neuropsychiatry and Clinical Neurosciences. 2011;23(2):Abstracts, p2.

54. Carton S, Thompson PJ, Duncan JS. Non-epileptic seizures: patients' understanding and reaction to the diagnosis and impact on outcome. Seizure. 2003;12(5):287-294.

55. Hall-Patch L, Brown R, House A, et al.; NEST collaborators. Acceptability and effectiveness of a strategy for the communication of the diagnosis of psychogenic nonepileptic seizures. Epilepsia 2010; 51(1): $70-78$

56. Voon V, Gallea C, Hattori N, Bruno M, Ekanayake V, Hallett M. The involuntary nature of conversion disorder. Neurology. 2010;74(3): 223-228.

57. Stone J, Zeman A, Simonotto E, et al. fMRI in patients with motor conversion symptoms and controls with simulated weakness. Psychosom Med. 2007;69(9):961-969.

58. Ward NS, Oakley DA, Frackowiak RS, Halligan PW. Differential brain activations during intentionally simulated and subjectively experienced paralysis. Cogn Neuropsychiatry. 2003;8(4):295-312.

59. Goldstein LH, Deale AC, Mitchell-O'Malley SJ, Toone BK, Mellers JD An evaluation of cognitive behavioral therapy as a treatment for dissociative seizures. Cogn Behav Neurol. 2004;17(1):41-49.

60. Goldstein LH, Chalder T, Chigwedere C, et al. Cognitive-behavioral therapy for psychogenic nonepileptic seizures: a pilot RCT. Neurology. 2010;74(24):1986-1994.

61. LaFrance WC Jr, Miller IW, Ryan CE, et al. Cognitive behavioral therapy for psychogenic nonepileptic seizures. Epilepsy Behav. 2009; 14(4):591-596.

62. Kuyk J, Siffels MC, Bakvis P, Swinkels WA. Psychological treatment of patients with psychogenic non-epileptic seizures: an outcome study. Seizure. 2008;17(7):595-603.

63. Gabbard GO. Basic principles of dynamic psychiatry. In: Gabbard GO. Psychodynamic Psychiatry in Clinical Practice. Washington, DC: American Psychiatric Publishing; 2005:3-30.

64. Mayor R, Howlett S, Grünewald R, Reuber M. Long-term outcome of brief augmented psychodynamic interpersonal therapy for psychogenic nonepileptic seizures: seizure control and health care utilization. Epilepsia. 2010;51(7):1169-1176.

65. Howlett S, Reuber M. An augmented model of brief psychodynamic interpersonal therapy for patients with nonepileptic seizures. Psychotherapy (Chic). 2009;46(1):125-138.

66. Barry JJ, Wittenberg D, Bullock KD, Michaels JB, Classen CC, Fisher RS Group therapy for patients with psychogenic nonepileptic seizures: a pilot study. Epilepsy Behav. 2008;13(4):624-629.

67. Prigatano GP, Stonnington CM, Fisher RS. Psychological factors in the genesis and management of nonepileptic seizures: clinical observations. Epilepsy Behav. 2002;3(4):343-349.

68. Zaroff CM, Myers L, Barr WB, Luciano D, Devinsky O. Group psychoeducation as treatment for psychological nonepileptic seizures. Epilepsy Behav. 2004;5(4):587-592.

69. LaFrance WC Jr, Keitner GI, Papandonatos GD, et al. Pilot pharmacologic randomized controlled trial for psychogenic nonepileptic seizures. Neurology. 2010;75(13):1166-1173.

70. Pintor L, Baillés E, Matrai S, et al. Efficiency of venlafaxine in patients with psychogenic nonepileptic seizures and anxiety and/or depressive disorders. J Neuropsychiatry Clin Neurosci. 2010;22(4):401-408.

71. Gross RA, Johnson KC. Levels of evidence: taking neurology to the next level. Neurology. 2009;72(1):8-10.

72. Quinn MC, Schofield MJ, Middleton W. Successful psychotherapy for psychogenic seizures in men. Psychother Res. Epub July 18, 2012.

73. Barry JJ, Atzman O, Morrell MJ. Discriminating between epileptic and nonepileptic events: the utility of hypnotic seizure induction. Epilepsia. 2000;41(1):81-84 
74. Khan AY, Baade L, Ablah E, McNerney V, Golewale MH, Liow K. Can hypnosis differentiate epileptic from nonepileptic events in the video/EEG monitoring unit? Data from a pilot study. Epilepsy Behav. 2009;15(3):314-317.

75. Kabat-Zinn J. Full Catastrophe Living: Using the Wisdom of your Body and Mind to Face Stress, Pain and Illness. New York: Delacorte; 1990.

76. Hayes S, Strosahl K, Wilson K. Acceptance and Commitment Therapy. New York: Guilford; 1999.

77. Linehan M. Cognitive-Behavioral Treatment of Borderline Personality Disorder. New York: Guilford; 1993.

78. Segal Z, Williams J, Teasdale J. Mindfulness-Based Cognitive Therapy for Depression: A New Approach to Preventing Relapse. New York: Guilford; 2002.

79. Shapiro SL, Carlson LE, Astin JA, Freedman B. Mechanisms of mindfulness. J Clin Psychol. 2006;62(3):373-386.

80. Baslet G, Hill J. Case report: brief mindfulness-based psychotherapeutic intervention during inpatient hospitalization in a patient with conversion and dissociation. Clin Case Studies. 2011;10(2):95-109.
81. Ogden P, Pain C, Fisher J. A sensorimotor approach to the treatment of trauma and dissociation. Psychiatr Clin North Am. 2006;29(1): 263-279.

82. Bakvis P, Roelofs K, Kuyk J, Edelbroek PM, Swinkels WA, Spinhoven P. Trauma, stress, and preconscious threat processing in patients with psychogenic nonepileptic seizures. Epilepsia. 2009;50(5): 1001-1011.

83. Goldstein LH, Mellers JDC. Ictal symptoms of anxiety, avoidance behavior, and dissociation in patients with dissociative seizures. J Neurol Neurosurg Psychiatry. 2006;77(5):616-621.

84. Kelley SDM, Benbadis S. Eye movement desensitization and reprocessing in the psychological treatment of trauma-based psychogenic nonepileptic seizures. Clin Psychol Psychother. 2007;14(2):134-144.

85. Van der Kruijs SJ, Bodde NM, Vaessen MJ, et al. Functional connectivity of dissociation in patients with psychogenic non-epileptic seizures. J Neurol Neurosurg Psychiatry. 2012;83(3):239-247.

\section{Publish your work in this journal}

Neuropsychiatric Disease and Treatment is an international, peerreviewed journal of clinical therapeutics and pharmacology focusing on concise rapid reporting of clinical or pre-clinical studies on a range of neuropsychiatric and neurological disorders. This journal is indexed on PubMed Central, the 'PsycINFO' database and CAS.
The manuscript management system is completely online and includes a very quick and fair peer-review system, which is all easy to use. Visit http://www.dovepress.com/testimonials.php to read real quotes from published authors.

\footnotetext{
Submit your manuscript here: http://www.dovepress.com/neuropsychiatric-disease-and-treatment-journal
} 